\title{
Bewertung regulatorischer Maßnahmen der Sektorenkopplung für den Einsatz von Power-to-Heat
}

\author{
Benjamin Grosse $^{1}$ (D) - Yannick Werner ${ }^{1}$ (D) - Denise Held ${ }^{2}$ (D) · Joschka Selinger ${ }^{2}$ Simon Schäfer-Stradowsky² (D) \\ Joachim Müller-Kirchenbauer'
}

Online publiziert: 13. Oktober 2020

(c) Springer Fachmedien Wiesbaden GmbH, ein Teil von Springer Nature 2020

\section{Zusammenfassung}

Die Kopplung der Sektoren Strom, Wärme, Gas und Verkehr stellt die nächste Stufe der Energiewende in Deutschland dar. Hierzu muss einerseits ein verbindlicher Rechtsrahmen geschaffen werden, andererseits müssen Geschäftsmodelle entstehen, welche auf Basis dieses Rechtsrahmen die Sektorenkopplung auch in die Praxis umsetzen. Aktuell bestehen jedoch verschiedene regulatorische Hemmnisse, welche einer solchen Praxisanwendung entgegenstehen und insbesondere die Integration von erneuerbarem Strom in den Wärmesektor (Power-to-Heat, PtH) behindern. In diesem Beitrag werden ausgehend von einer Darstellung des regulatorischen Status Quo auf Basis eines Literaturreviews 21 regulatorische Maßnahmen untersucht und miteinander verglichen. Für vier ausgewählte Maßnahmen, die einerseits auf eine Reduktion der Netzentgelte, der EEG-Umlage und andererseits auf die Einführung einer sektorenübergreifenden $\mathrm{CO}_{2}$ - $\mathrm{Bepreisung}_{\text {zielen, }}$ wird eine detaillierte einzel-ökonomische, rechtliche und systemische Analyse am Fallbeispiel einer PtH-Anlage in einem Fernwärmnetz durchgeführt. Es zeigt sich, dass eine PtH-Anlage im Status Quo nicht betriebswirtschaftlich auskömmlich zu betreiben ist. Darüber hinaus scheint die Einführung einer sektorenübergreifenden $\mathrm{CO}_{2}$-Bepreisung aus allen betrachteten Perspektiven besonders vorteilhaft. Jedoch sind weitere Maßnahmen notwendig und erscheinen rechtlich und systemisch möglich, um unter den getroffenen Annahmen die Investition in eine PtH-Anlage ökonomisch rentabel zu machen.

\section{Evaluation of Various Regulatory Policies for the Use of Power-to-Heat}

\begin{abstract}
The coupling of the sectors electricity, heat, gas, and mobility represents the next stage in the German Energiewende. For this purpose, a binding legal framework must be created on the one hand, and on the other hand, business models must be developed, which enable sector coupling based on that legal framework. Currently, however, there are various obstacles especially in the area of integrating renewable electricity into the heating sector (Power-to-Heat, PtH). Starting with a description of the regulatory status quo, this article analyzes and compares 21 different regulatory policies based on a literature review. For four selected policies, which on the one hand aim at a reduction of the network charges or the EEG surcharge and on the other hand at introducing $\mathrm{CO}_{2}$ pricing across all sectors, a detailed microeconomic, legal and systemic analysis is carried out using the example of a PtH plant that feeds into a district heating network. We show that it is not possible to run the $\mathrm{PtH}$ plant economically profitable manner from a company viewpoint. Furthermore, from all analyzed perspectives, the introduction of a cross sectoral $\mathrm{CO}_{2}$ pricing seems to most beneficial. Nevertheless, further measures, that seem to be legally and systemically possible, are necessary to make the investment into a PtH plant economically profitable under the given assumptions.
\end{abstract}

Benjamin Grosse

grosse@tu-berlin.de

1 Institut für Technologie und Management (ITM), Fachgebiet Energie- und Ressourcenmanagement, Technische Universität Berlin, Fraunhoferstr. 33-36, 10587 Berlin, Deutschland

2 IKEM - Institut für Klimaschutz, Energie und Mobilität e. V., Berlin, Deutschland

\section{Einleitung}

Die Bundesregierung hat sich mit dem Klimaschutzplan 2050 ambitionierte Ziele gesetzt und strebt ein treibhausgasneutrales Gesamtsystem bis 2050 an (BMU 2019). Um dieses Ziel zu erreichen, ist eine schnelle Defossilisierung des Wärmesektors, der etwa die Hälfte des gesamten Endenergieverbrauchs in Deutschland ausmacht (UBA 2020), 
unerlässlich. Der Anteil erneuerbarer Energien am Wärmeverbrauch stagniert jedoch seit 2012 (UBA 2020). Eine Möglichkeit, diesen Anteil zu erhöhen und die Wärmewende als Teil der Energiewende weiter voranzutreiben, ist der Einsatz von erneuerbarem Strom im Wärmesektor (Sektorenkopplung). Power-to-Heat kommt dabei eine Schlüsselfunktion (Fraunhofer IWES/IBP 2017) vor allem im Bereich der Fernwärmenetze und Prozesswärmebereitstellung (Fraunhofer ISE 2020) zu. Das technische Potential von Power-to-Heat-Technologien im Energiesystem der Zukunft ist vermutlich sehr groß (Gils et al. 2016), weshalb diese Sektorenkopplung in den Fokus des politischen und wissenschaftlichen Energiewendediskurses gerückt ist.

Als Power-to-Heat $(\mathrm{PtH})$ wird allgemein eine Technologie bezeichnet, die Strom aus weitüberwiegend erneuerbarer Erzeugung direkt oder indirekt in Wärme wandelt (Wietschel et al. 2019). Für PtH-Technologien werden zwei zentrale Beiträge für die Dekarbonisierung des Energiesystems identifiziert: Die PtH-Technologien als Baustein ,grüner" Wärmeversorgung durch die Umwandlung von Strom aus erneuerbaren Energien in emissionsfreie Wärme (BCG und Prognos AG 2018, S. 59) und die Nutzung von „Überschussstrom“, insbesondere in Verbindung mit einem Wärmespeicher, als kostengünstige Flexibilitätsoption für den Stromsektor (Fraunhofer IWES et al. 2014). Durch aktuelle regulatorische Vorgaben wie die Reduktion der Netzentgelte bei atypischer Netznutzung oder besonders intensiver Netznutzung ( $\$ 19$ Stromnetzentgeltverordnung (StromNEV)) wird die Nutzung dieser Flexibilität allerdings erschwert.

Der potenzielle Beitrag von $\mathrm{PtH}$, speziell im MegawattBereich für Fernwärmenetze, für ein defossilisiertes Energiesystem wird im regulatorischen Rahmen daher bislang nur ungenügend berücksichtigt. Aufgrund regulatorischer Hemmnisse (Hilpert 2015; Wietschel et al. 2019), insbesondere der Kostenbelastung für den Strombezug durch staatlich veranlasste Preisbestandteile, ist der Einsatz von Groß$\mathrm{PtH}$-Anlagen derzeit oftmals nicht wirtschaftlich (Drünert et al. 2019). Hinzu kommen die gesetzlichen Regelungen für die Nutzung der erzeugten Wärme, die ein weiteres Hemmnis für den Einsatz von PtH-Technologien darstellen.

Der vorliegende Beitrag ergänzt die bestehende Literatur durch eine interdisziplinäre Betrachtung von ausgewählten regulatorischen Maßnahmen, welche das Ziel haben diese Hemmnisse abzubauen. Hierzu werden diese aus rechtlichregulatorischer Sicht bzw. hinsichtlich ihrer Auswirkungen aus einzel-ökonomischer und systemischer Perspektive analysiert. Somit wird eine fundierte Empfehlung der Rangfolge zur Umsetzung betrachteter Maßnahmen gegeben. Die Arbeit beginnt zunächst mit einer Zusammenfassung des aktuellen Rechtsrahmens. Anschließend werden Maßnahmen zum Abbau regulatorischer Hemmnisse auf Basis eines Literaturreviews analysiert und ausgewählt. Diese werden im Anschluss näher analysiert. Ein Fazit schließt den Beitrag ab.

\section{Aktueller Rechtsrahmen}

Der Rechtsrahmen berücksichtigt PtH-Technologien nur punktuell, speziell auf $\mathrm{PtH}$ zugeschnittene Regelungen existieren nicht (Doderer et al. 2018). Der potenzielle Beitrag von PtH zur Dekarbonisierung des Energiesystems wird durch den aktuellen Rechtsrahmen nicht abgebildet. Dieser lässt sich grundsätzlich in Regelungen bezüglich des Einsatzstoffs (Strom), des Produkts (Wärme) und der Anlagen und Infrastrukturen unterteilen. Das derzeit wesentliche regulatorische Hemmnis für einen wirtschaftlichen Betrieb von PtH-Anlagen ist die Kostenbelastung durch hohe Strombezugskosten (Drünert et al. 2019). Daher wird der aktuelle Rechtsrahmen im Folgenden mit einem Schwerpunkt auf dem Strombezug analysiert.

Vereinfacht gesagt fallen bei Strombezug aus dem Netz der allgemeinen Versorgung durch PtH-Anlagen grundsätzlich alle damit zusammenhängenden Entgelte, Umlagen und Abgaben an, da PtH-Anlagen als Letztverbraucher i.S.d. $\S 3 \mathrm{Nr} .25$ Energiewirtschaftsgesetz (EnWG), § 3 Nr. 33 Erneuerbare-Energien-Gesetz (EEG 2017) angesehen werden. Die bestehenden gesetzlichen Ausnahmetatbestände greifen regelmäßig nicht. Dadurch fallen erhebliche Stromnebenkosten an, die sich negativ auf die Wirtschaftlichkeit von PtH-Anlagen auswirken. Die größten Kostenblöcke bei den staatlich veranlassten Preisbestandteilen sind die Netzentgelte (auch Netznutzungsentgelte (NNE)) und die EEGUmlage, die jeweils über $20 \%$ des Haushaltskundenstrompreises in 2019 ausmachten (BNetzA und BKartA 2019, S. 9).

Die Systematik der Netzentgelte kennt zahlreiche Ausnahmetatbestände, bei deren Vorliegen das Netzentgelt gar nicht oder nur in reduzierter Höhe anfällt. Für PtHAnlagen sind diese jedoch regelmäßig entweder nicht einschlägig oder stehen dem systemdienlichen Einsatz der PtH-Anlage entgegen. So kommt die befristete Befreiung von den Netzentgelten nach $\S 118$ Abs. 6 EnWG (,Speicherprivileg") für $\mathrm{PtH}$-Anlagen nicht in Betracht, da dieser die zeitlich verzögerte Rückverstromung in dasselbe Netz voraussetzt. Die Regel ist jedoch gerade der Verbrauch der Wärme. Auch die Netzentgeltreduzierung nach $\S 19$ Abs. 2 Stromnetzentgeltverordnung (StromNEV) kommt bei einem netz- und systemdienlichen Anlagenbetrieb nicht in Betracht. Gem. § 19 Abs. 2 S. 1 StromNEV können die Netzentgelte bei einer atypischen Netznutzung der Anlage auf bis zu $20 \%$ des veröffentlichten Netzentgeltes (individuelles Netzentgelt) nach vorheriger Genehmigung durch die Bundesnetzagentur (BNetzA) reduziert werden. Diese ist bei PtH-Anlagen zwar grundsätzlich möglich, 
die atypische Netznutzung setzt jedoch voraus, dass der Höchstlastbeitrag vorhersehbar erheblich von der zeitgleichen Jahreshöchstlast aller Entnahmen abweicht. Dies kann bei einem flexiblen, netzdienlichen Anlageneinsatz nicht gewährleistet werden, da bei Strombezug außerhalb der Hochlastzeitfenster der Verlust der Reduzierung droht. Gleiches gilt für die Netzentgeltreduzierung nach $§ 19$ Abs. 2 Satz 2 StromNEV, welche 7000 Vollbenutzungsstunden voraussetzt. Schließlich kommt eine Netzentgeltreduzierung nach $\S 14 \mathrm{a}$ EnWG in Betracht, wenn der Anlagenbetreiber dem Netzbetreiber eine netzdienliche Steuerung der Anlage ermöglicht. Diese Vorschrift kann bislang noch nicht sinnvoll oder flächendeckend angewendet werden, da sie zunächst durch eine Rechtsverordnung konkretisiert werden müsste. Der Erlass dieser Rechtsverordnung steht seit Einführung der Vorschrift im Jahr 2011 (BGBl. 2011 I, S. 1554) aus. Neben den Netzentgelten fallen weitere netzentgeltbezogene Preisbestandteile an: die KWK-Umlage, die § 19 Abs. 2 StromNEV-Umlage und die Offshore-Umlage. Diese netzentgeltgewälzten Belastungen entfallen nicht automatisch, wenn ein Befreiungstatbestand von den Netzentgelten vorliegt (BGH 2017).

Ähnlich verhält es sich bei der EEG-Umlage. Das EEG 2017 sowie das Anfang 2017 reformierte Kraft-WärmeKopplungsgesetz (KWKG) enthalten zahlreiche Neuerungen und Ansatzpunkte zum Abbau der damit verbundenen Kostenbelastung. Diese knüpfen jedoch überwiegend an die Rückverstromung des aus dem Netz entnommenen Stroms an, sodass PtH-Anlagen davon in der Regel nicht profitieren können.

Ein weiterer erheblicher Kostenfaktor für den Strombezug von PtH-Anlagen ist die Stromsteuer. Die Stromsteuer entsteht gem. $\S 5$ Abs. 1 S. 1 Stromsteuergesetz (StromStG) sowohl bei Entnahme aus dem Netz als auch bei Selbstverbrauch. Für PtH-Anlagen, die in Unternehmen des produzierenden Gewerbes genutzt werden, kommt eine anteilige Entlastung von der Stromsteuer nach § 9b StromStG in Betracht. Andere Entlastungstatbestände greifen regelmäßig nicht, sodass im Regelfall die volle Stromsteuer zu entrichten ist.

Neben den hohen Strombezugskosten bestehen weitere Hemmnisse für die Marktdurchdringung von PtH-Anlagen. So besteht kein kohärenter Rechtsrahmen für die Förderung erneuerbarer Wärme und auch der Netzanschluss von PtHAnlagen ist z.T. mit erheblichen Kosten verbunden, da die Netzanschlusskosten durch Baukostenzuschüsse (BKZ) auf den Anlagenbetreiber umgelegt werden können.

\section{Qualitative Maßnahmenauswahl mittels Literaturreview}

Prinzipiell besteht eine breite Varietät von regulatorischen Maßnahmen. Daher wird die Auswahl der zu bewertenden Maßnahmen in einem zweistufigen Verfahren durchgeführt, welches im Folgenden näher beschrieben wird.

\subsection{Verfahren zur Maßnahmenauswahl}

Zunächst werden aus der aktuellen Literatur relevante Maßnahmen aufgenommen. Aufgrund der Vielzahl existierender Maßnahmen werden diese anhand folgender Kriterien ausgewählt: Solche, die (a) eine positive ökonomische Auswirkung auf $\mathrm{PtH}-$ Anlagen erwarten lassen und entweder (b) kurzfristig bis mittelfristig umsetzbar erscheinen, oder (c) aktuell besonders im Gespräch sind (d.h. in mindestens zwei der analysierten Studien genannt wurden) sowie (d) deren rechtliche Einführung prinzipiell möglich scheint.

In einem zweiten Schritt werden einzelne Maßnahmen für eine spezifischere Evaluation ausgewählt. Hierzu werden die Maßnahmen qualitativ hinsichtlich der Höhe des zu erwartenden (positiven) Einflusses auf die Wirtschaftlichkeit von PtH-Geschäftsmodellen (PtH-GM), sowie der (notwendigen) regulatorischen Eingriffstiefe ${ }^{1}$ paarweise miteinander verglichen und eine Null-Eins-Metrik angewandt. Eine Maßnahme wird dabei hinsichtlich der Eingriffstiefe mit eins bewertet, wenn eine größere regulatorische Eingriffstiefe vorliegt, als bei der Vergleichsmaßnahme $(0,5$ bei gleicher und 0 bei geringerer). Dies bedeutet, dass mehr bzw. weiterführende Gesetzesänderungen notwendig sind und/ oder mehr Stakeholder in den Prozess einzubeziehen sind. Hinsichtlich der Höhe des zu erwartenden Einflusses auf die Wirtschaftlichkeit von PtH-GM wird eine Abschätzung vorgenommen, wie groß die zu erwartenden ökonomischen Vorteile einer Maßnahme gegenüber einer anderen sind. Hierbei wird im paarweisen Vergleich die Maßnahmen mit eins bewertet, die relativ die größere Erlöserhöhung erwarten lässt.

Im Anschluss werden die Ergebnisse des paarweisen Vergleichs in eine Matrix überführt. So können die Maßnahmen identifiziert werden, welche überdurchschnittliche ökonomische Ergebnisse erwarten lassen und gleichzeitig der Aufwand für die Einführung abgeschätzt werden, welcher als zusätzliches Auswahlkriterium bei gleich wirkenden Maßnahmen genutzt wird. Um eine eindeutige Aussage hinsichtlich der Ursache-Wirkungs-Relation zu gewährleisten, wird die Auswahl dabei auf solche Maßnahmen eingeschränkt, welche durch einen spezifischen Eingriff wirken, also keine Kombination von Maßnahmen darstellen.

\footnotetext{
${ }^{1}$ Bezüglich regulatorischer Eingriffstiefe vgl. auch Jansen und Sager-
} Klauß (2017). 
Tab. 1 Übersicht regulatorischer Maßnahmen

\begin{tabular}{|c|c|c|c|}
\hline $\mathrm{Nr}$ & Ziel & Inhalt & Frist \\
\hline 1 & EEG-Dyn. ${ }^{\mathrm{a}, \mathrm{e}, \mathrm{f}, \mathrm{g}, \mathrm{j}}$ & Dynamisierung der EEG-Umlage in Abhängigkeit des Börsenstrompreises & $\mathrm{m}$ \\
\hline 2 & EEG-Dyn. ${ }^{\mathrm{a}, \mathrm{f}}$ & Dynamisierung der EEG-Umlage gemäß der Netzsituation & $\mathrm{m}$ \\
\hline 3 & EEG-Red. ${ }^{\mathrm{d}}$ & Erweiterung der Umlagereduzierung des $§ 611$ EEG für Stromspeicher auf PtH & $\mathrm{k}$ \\
\hline 4 & EEG-Red..$^{\mathrm{f} h}$ & $\begin{array}{l}\text { Wälzung der EEG-Umlage über mehrere Sektoren, d.h. ein Einbezug der durch die Sekto- } \\
\text { renkopplung profitierenden Sektoren Verkehr und Wärme }\end{array}$ & $\mathrm{m}$ \\
\hline 5 & EEG-Red. ${ }^{\mathrm{a}}$ & $\begin{array}{l}\text { Wälzung der EEG-Umlage über mehrere Sektoren, Einbezug der ausschließlichen Ver- } \\
\text { brauchsanteile in weiteren Sektoren (Verkehr und Wärme) }\end{array}$ & $\mathrm{m}$ \\
\hline 6 & EEG-Red. ${ }^{\mathrm{a}, \mathrm{f}}$ & Fondsfinanzierung des EEG & $\mathrm{k}$ \\
\hline 7 & EEG-Red. ${ }^{\mathrm{f}}$ & Reduktion von Ausnahmeregelungen des EEGs (Eigenverbrauch, BesAR) & $\mathrm{k}$ \\
\hline 8 & Inv.-Red. ${ }^{\mathrm{c}, \mathrm{d}}$ & BKZ bei geeigneter Standortwahl für PtH-Anlagenbetreiber & $\mathrm{k}$ \\
\hline 9 & Inv.-Red. ${ }^{\mathrm{d}, \mathrm{l}}$ & $\begin{array}{l}\text { Anpassung des } \S 13 \text { Abs. 6a EnWG bzw. der Rechtsverordnungen zu Abschaltbaren Lasten } \\
\text { sowie eine Ergänzung des Verfahrens zu Zuschaltbaren Lasten }\end{array}$ & $\mathrm{k}$ \\
\hline 10 & NNE-Dyn. ${ }^{j}$ & Dynamisierung der Netzentgelte gemäß der Netzsituation & $\mathrm{m}$ \\
\hline 11 & NNE-Red. ${ }^{\mathrm{d}, \mathrm{l}}$ & $\begin{array}{l}\text { Erweiterung der Netzentgeltreduzierung nach } \S 14 \mathrm{a} \text { EnWG bzw. Konkretisierung der Vor- } \\
\text { schrift für System- und Marktdienlichkeit sowie für höhere Spannungsebenen }\end{array}$ & $\mathrm{k}$ \\
\hline 12 & NNE-Red. ${ }^{\mathrm{d}, \mathrm{l}}$ & Erweiterung Netzentgeltbefreiung nach $\S 118$ EnWG i.S. v. PtH ohne Rückverstromung & $\mathrm{k}$ \\
\hline 13 & NNE-Red..$^{\mathrm{d}, \mathrm{l}}$ & $\begin{array}{l}\text { Anpassung des } \S 19 \text { Abs. } 2 \text { StromNEV, um markt-, system- und netzdienliches Verhalten } \\
\text { unter Reduktion der Netznutzungsentgelte zu ermöglichen }\end{array}$ & $\mathrm{m}$ \\
\hline 14 & Produkt $^{\mathrm{d}}$ & Anerkennung von PtH als erneuerbare Wärme zwecks Veräußerungsoptionen & $\mathrm{k}$ \\
\hline 15 & Produkt ${ }^{\mathrm{d}}$ & Anpassung der Definition des PEF im Sinne der EnEV & $\mathrm{k}$ \\
\hline 16 & StromSt-Dyn. ${ }^{\mathrm{d}}$ & Aufkommensneutrale Dynamisierung der StromSt in Abhängigkeit des Börsenstrompreises & $\mathrm{m}$ \\
\hline 17 & StromSt-Red. ${ }^{\mathrm{d}, \mathrm{l}}$ & Erweiterung der Steuerentlastung nach $\S 9$ StromStG um PtH als Ausnahmetatbestand & $\mathrm{m}$ \\
\hline 18 & $\begin{array}{l}\text { Neue } \\
\text { Zahlung }, \text { c,b,f,i,k }\end{array}$ & Sektorenübergreifende $\mathrm{CO}_{2}$-Bepreisung & $\mathrm{m}$ \\
\hline 19 & Neue Zahlung ${ }^{\mathrm{c}, \mathrm{j}}$ & Ersatz von SAU durch sektorenübergreifende $\mathrm{CO}_{2}$-Bepreisung & - \\
\hline 20 & Neue Zahlung ${ }^{\mathrm{a}, \mathrm{b}}$ & $\begin{array}{l}\text { Umlage auf alle Energieträger zur Förderung jeglicher EE-Integration in einem beliebigen } \\
\text { Anwendungssektor (EE-Wärme/EE-Strom/EE-Verkehr) }\end{array}$ & - \\
\hline 21 & Neue Zahlung ${ }^{\mathrm{j}}$ & Netzentgelte in Infrastrukturumlage überführen & $\mathrm{m}$ \\
\hline
\end{tabular}

Frist: $k$ kurzfristig umsetzbar, $m$ mittelfristig umsetzbar, - unklar

BesAR Besondere Ausgleichsregelung, BKZ Baukostenzuschüsse, EE Erneuerbare Energien, EEG Erneuerbaren-Energien-Gesetz,

EnEV Energieeinsparverordnung, Inv. Investition, $N N E$ Netz(nutzungs)entgelte, PEF Primärenergiefaktor, SAU Steuern, Abgaben und Umlagen, StromNEV Stromnetzentgeltverordnung, StromSt Stromsteuergesetz

${ }^{\mathrm{a}}$ Agora Energiewende (2017)

${ }^{\mathrm{b}}$ Agora Energiewende (2018)

${ }^{\mathrm{c}}$ Dertinger und Schill (2019)

dDoderer et al. (2018)

${ }^{\text {eEcofys }}$ (2014)

${ }^{\mathrm{f}}$ Fiedler et al. (2017)

gFrontier Economics und BET (2016)

${ }^{\text {h}}$ Gährs et al. (2017)

i'W (2018)

jJansen und Sager-Klauß (2017)

${ }^{\mathrm{k}}$ Reuster et al. (2017)

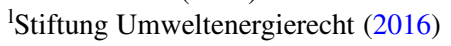

\subsection{Ergebnisse}

In der ersten Stufe der Maßnahmenauswahl werden 21 regulatorische Maßnahmen aus der einschlägigen Literatur übernommen, welche in Tab. 1 aufgelistet sind. Hierbei ist einerseits eine Kurzangabe hinsichtlich des Zieles der Maßnahme und eine Beschreibung gegeben. Darüber hinaus ist vermerkt, ob eine Umsetzung kurz- $(\mathrm{k})$ oder mittelfristig (m) möglich erscheint.
Prinzipiell kann zwischen solchen Maßnahmen unterschieden werden, die auf eine Veränderung von Steuern, Abgaben oder Umlagen zielen, sei es eine Reduktion (3-7, $10-12,16)$ oder Dynamisierung $(1,2,15,20)$ und solchen, die auf einen Zuschuss zur Investition zielen $(8,9)$. Ferner betreffen zwei Maßnahmen $(13,14)$ eine Anpassung der Rechtsnormen hinsichtlich der Anerkennung der Produkteigenschaften als Erneuerbare Wärme. Vier Maßnahmen $(17-19,21)$ grenzen sich insofern ab, als dass die- 
Abb. 1 Gegenüberstellung der Maßnahmen

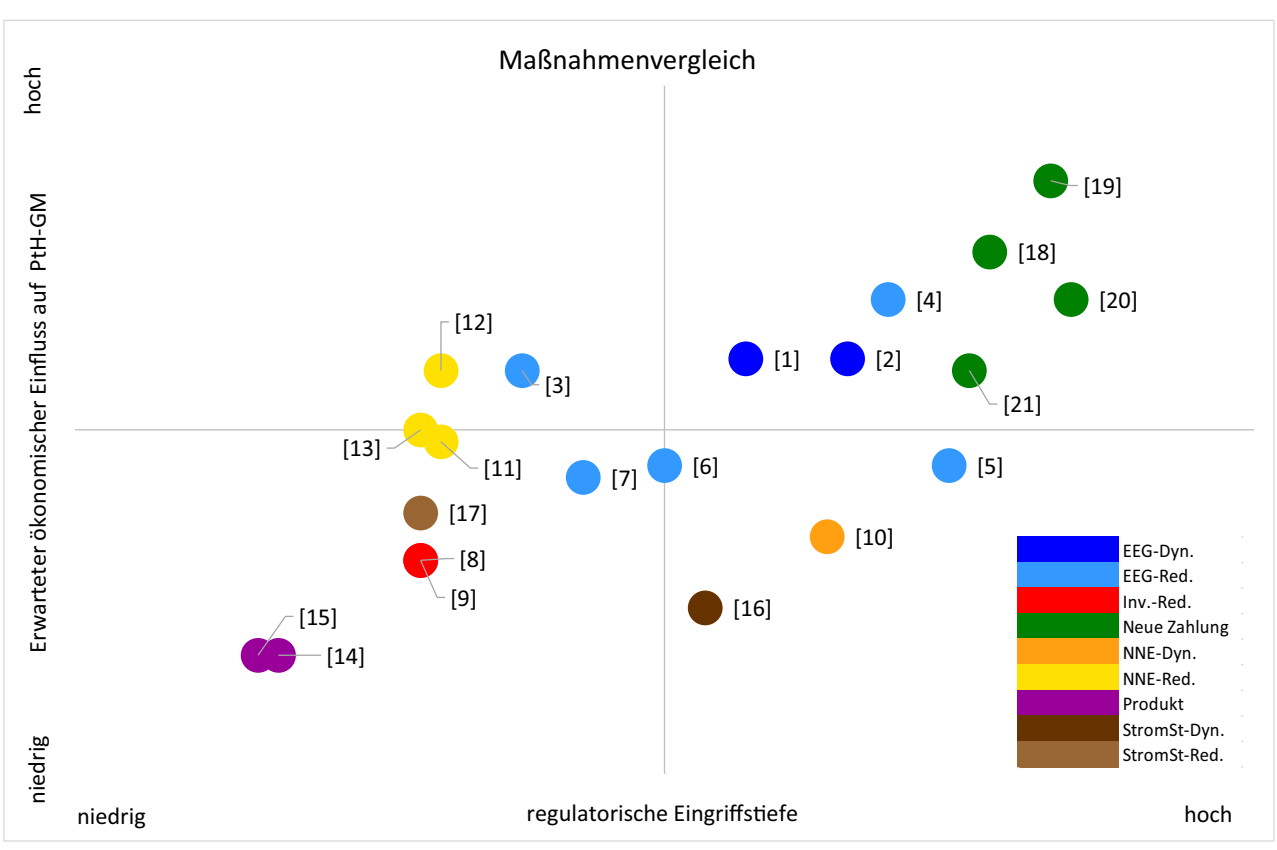

se die (breite) Einführung einer neuen Zahlung fordern. Zwei davon zielen dabei auf die Einführung einer sektorenübergreifenden $\mathrm{CO}_{2}$-Abgabe, wobei diese einerseits als Ergänzung, andererseits als Ersatz der bestehenden Steuern, Abgaben, Umlagen und Entgelte (SAUE) aufgefasst wird. Ein weiterer Ansatz stellt das Ziel der Einführung einer „Energiewendeumlage“ dar, welche sämtliche Förderung von Erneuerbaren Energien in allen Sektoren zusammen gegenfinanzieren soll, ein andere die Überführung der Netzentgelte in eine Infrastrukturabgabe.

Der paarweise Vergleich der Maßnahmen hinsichtlich ihres zu erwartenden ökonomischen Einflusses auf PtHGeschäftsmodelle, sowie der regulatorischen Eingriffstiefe (siehe Abb. 1) zeigt, dass besonders neue Zahlungen im Sinne einer sektorenübergreifenden $\mathrm{CO}_{2}$-Bepreisung (18 und 19) ökonomisch interessant zu bewerten sind. Dies begründet sich durch den Ausgleich von Kostennachteilen gegenüber anderen $\left(\mathrm{CO}_{2}\right.$ intensiveren) Technologien, welche durch externe Kosten induziert werden. Auf die $\mathrm{CO}_{2}$ preisbasierten Maßnahmen folgt aus ökonomischer Sicht die Reduktion der EEG-Umlage (4), sowie die Einführung einer sektorenübergreifenden EE-Abgabe (20). Erstere ist hier leicht besser zu bewerten, da es sich ausschließlich um die Wälzung der EEG-Kosten handelt, während im zweiten Fall (20) möglicherweise weitere Kosten gewälzt werden müssen und somit die spezifischen SAUE für die PtHAnlage höher liegen können als im ersten Fall (4). Überdurchschnittliche positive Auswirkungen auf die PtH-GM lässt sich ebenfalls von den Maßnahmen 1 und 2 erwarten, wobei die Höhe von den Vollbenutzungsstunden abhängig ist. Etwas geringere allerdings untereinander vergleichbare ökonomische Verbesserungen für PtH-GM sollten weiter- hin die Maßnahmen 12, 3 und 21 erreichen, wobei sich die regulatorische Eingriffstiefe zwischen ihnen deutlich unterscheidet.

Da Maßnahme 19, wie auch Maßnahme 20, als Kombination verschiedener einzelner Maßnahmen aufzufassen ist, werden diese nicht weiter betrachtet (siehe Abschn. 3.1). Auch wenn bei Maßnahme 18 eine hohe regulatorische Eingriffstiefe zu erwarten ist, wird Maßnahme 18 als aussichtsreiche ökonomische Maßnahme übernommen. Gleiches gilt für Maßnahme 4. Da sich Maßnahmen 1 und 2 sehr ähneln, wird aufgrund der geringeren Eingriffstiefe lediglich Maßnahme 1 in der weiteren Analyse inkludiert. Von den Maßnahmen 3, 12 und 21 wird ausschließlich 12 übernommen. Maßnahme 3 wird ausgeschlossen, da mit Maßnahme 4 bereits eine Reduktion der EEG-Umlage analysiert wird, sowie 3 und 12 ähnliche Auswirkungen auf PtH-GM erwarten lassen, wobei 12 die geringere Eingriffstiefe aufweist. Letzteres gilt verstärkt gegenüber Maßnahme 21, wobei zusätzlich Maßnahmen 12 und 21 aus einzelwirtschaftlicher Perspektive die gleiche Auswirkung (NNE-Reduktion) erreichen sollten. Daher wird 21 ausgeschlossen. Die weiteren aufgeführten Maßnahmen werden in der folgenden Analyse nicht betrachtet, da sie entweder ökonomisch unvorteilhafter erscheinen oder zu stark in bestehende Strukturen eingreifen. Insgesamt werden somit Maßnahmen 1, 4, 12 und 18 in die weitere Analyse übernommen.

\subsection{3 Spezifizierung der ausgewählten Maßnahmen}

Insgesamt werden vier Maßnahmen näher untersucht. (a) eine Reduktion der Netzentgelte (Maßnahme 12), (b) eine 
sektorenübergreifende Wälzung der EEG-Umlage (Maßnahme 4), (c) die Dynamisierung der EEG-Umlage (Maßnahme 1) und abschließend (d) die Einführung einer sektorenübergreifenden $\mathrm{CO}_{2}$-Bepreisung (Maßnahme 18). Für die weitere Bewertung der Maßnahmen wurde auf die o.g. Literatur zurückgegriffen und die folgenden Ausgestaltungen der Maßnahmen genutzt.

a) Für die Netzentgeltreduktion/-befreiung werden zwei verschiedene Optionen analysiert:

- (a1) Es werden keine Arbeitsnetzentgelte für Stunden mit negativen Börsenstrompreis fällig.

- (a2) Es werden keine Netzentgelte (Leistungs- und Arbeits-) für den Betrieb der PtH-Anlage fällig.

b) Die Berechnung für die sektorenübergreifende Wälzung der EEG-Umlage wird aus Gährs et al. (2017; Varianten (b) und (d)) übernommen und in Relation zum BasisEEG-Pfad (entnommen aus Haller 2020) fortgeschrieben, wobei zwei Szenarien unterschieden werden:

- (b1) Reduktion der Umlage bei Einbezug des Wärmesektors $30,32 € / \mathrm{MW}_{\mathrm{el}} \mathrm{h}$.

- (b2) Reduktion der Umlage bei Einbezug von Wärmeund Verkehrssektor 21,66€/MW el h.

c) Die Dynamisierung der EEG-Umlage lehnt sich an die Arbeit von Ecofys (2014) an, wobei eine Dynamisierung anhand des Day-Ahead-Strompreises und der an der EPEX SPOT gehandelten Strommengen des Jahres 2017 aufkommensneutral durchgeführt wird. ${ }^{2}$

d) Für die Einführung eines sektorenübergreifenden $\mathrm{CO}_{2}$ Preises wird zunächst auf die für Deutschland beschlossenen Preise bis 2025 (Bundesregierung 2019a) zurückgegriffen und im Folgenden der mittlere Preispfad von Edenhofer et al. (2019) gewählt.

\section{Vorgehen}

Die differenzierte Analyse der Maßnahmen (siehe Abschn. 3.3) wird zunächst aus ökonomischer, anschließend aus rechtlicher und zuletzt aus Verteilungsgerechtigkeitssicht durchgeführt. Als Fallbeispiel dient eine Megawatt-PtH-Anlage, welche in das bestehende Fernwärmenetz eines Stadtwerks integriert wird und dort einen Teil der Wärmebereitstellung substituieren soll. Dabei wird angenommen, dass im Status quo die Wärme- (und Strom-) versorgung über ein bestehendes Erdgas-Blockheizkraftwerk (Erdgas-BHKW) erfolgt. Abb. 2 gibt eine Übersicht über dem weiteren Aufbau der Analyse.

\footnotetext{
${ }^{2}$ Hierbei wird ein Multiplikator so bestimmt, dass die dynamisierte EEG-Umlage minimal $0 € / \mathrm{MW}_{\text {el }}$ und maximal das Zweifache der EEG-Umlage im Jahr 2017, 137,6€/MW elh beträgt. Dies ergab einen Multiplikator in Höhe von 2,09, welcher im Folgenden für alle Jahre des Investitionszeitraums verwendet wird.
}

\section{1 Ökonomische Analyse}

Um den aktuellen Status-quo des Fallbeispiels (und vieler Stadtwerke) aus ökonomischer Sicht möglichst adäquat abzubilden, wird im Folgenden bei der Investitionsrechnung ein Differenzkapitalwert unter Einbezug der Zahlungsströme des bestehenden BHKWs berechnet. Dabei werden sowohl die vermiedenen Kosten für den Erdgaseinsatz als auch die entgangenen Erlöse für den Verkauf des erzeugten Stroms zum Spot-Strompreis berücksichtigt. Weitere Ausgaben für Betrieb und Wartung des BHKWs werden jedoch nicht einbezogen, da diese vermutlich weiterhin anfallen, wenn die Wärmebereitstellung in Stunden, in denen der Betrieb der PtH Anlage sehr teuer ist, durch das BHKW erfolgen soll. Dies stellt für die Investition in eine PtHAnlage vermutlichen den schlechtesten Fall, für viele Erzeuger allerdings ein durchaus realistisches Szenario, dar. Ferner wird angenommen, dass die Jahresmindestlast des existierenden Fernwärmenetzes stehts über der Wärmeleistung der PtH-Anlage liegt. Daher kann unterstellt werden, dass sie lediglich zu den günstigsten Zeitpunkten und bei voller Leistung betrieben werden kann (vergleiche Drünert et al. 2019).

Um einen besseren Vergleich der verschiedenen Maßnahmen zu ermöglichen, wird zu nächst ein Basisszenario definiert, welches den aktuellen Stand der Regulierung abbildet. Anschließend werden die ausgewählten Maßnahmen hinsichtlich ihrer ökonomischen Auswirkung einzeln analysiert und verglichen. Hierzu werden zunächst durch das power market model energy and resoures (POMMES) ${ }^{3}$ stündlich aufgelöste Strompreiszeitreihen für den vorgegeben $\mathrm{CO}_{2}$-Preispfad bereitgestellt. Dabei werden einzelne Stützjahre berechnet und zwischen diesen wird linear interpoliert (siehe Tab. 2). Im Anschluss werden die ermittelten Spot-Strompreise um die relevanten weiteren Strompreisbestandteile für die PtH-Anlage beaufschlagt. Hierbei werden die Abgaben den o.g. Maßnahmen entsprechend angepasst. Abschließend werden Jahresmittelwerte der Strombezugskosten der PtH-Anlagen (in Abhängigkeit der vorgegebe-

\footnotetext{
${ }_{3}$ POMMES ist ein bottom-up Kostenminimierungsmodell, welches auf Basis fundamentaler, technischer und ökonomischer Parameter den deutschen Strommarkt abbildet. Dies erfolgt unter Annahme vollständiger Informationen sowie einem ausschließlichen Handel aller Strommengen am simulierten Day-Ahead Markt. Es basiert auf dem open energy modelling framework (oemof) (Hilpert et al. 2018) und kann als reines Dispatch- sowie als integriertes Investment- und Dispatchmodell verwendet werden. In dieser Analyse wurde das reine Dispatchmodell verwendet, da eine möglichst genaue stündliche Auflösung der Strompreise im Mittelpunkt stand. Im Modell werden Deutschland mit einem anlagenscharfen Kraftwerkspark, sowie angrenzende Staaten mit einem aggregierten Kraftwerkspark marktzonenscharf abgebildet. Die zugehörigen Übertragungskapazitäten werden auf Basis zeitlich variabler Net Transfer Capacities (NTCs) modelliert (Ghosh et al. 2019).
} 
Abb. 2 Aufbau der detaillierten Analyse

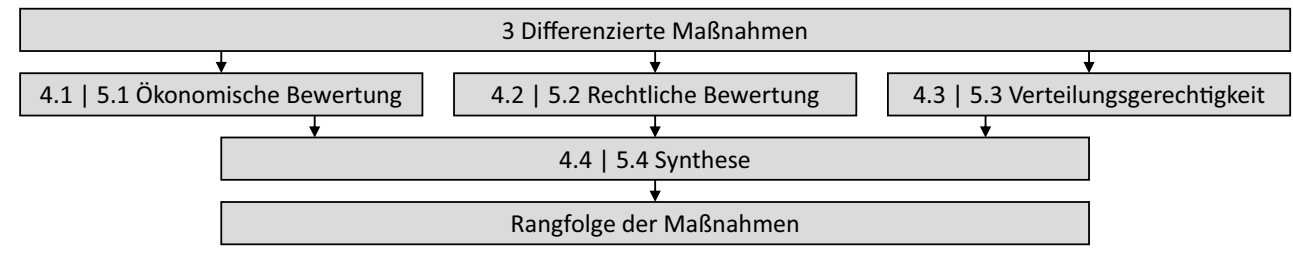

Tab. 2 Ergebnisse der Dispatchmodellierung für verschiedene Stützjahre mit dem Strommarktmodell POMMES. (Entwicklung Kraftwerkspark basierend auf BCG und Prognos AG 2018)

\begin{tabular}{|c|c|c|c|c|c|c|}
\hline & 2017 & 2020 & 2025 & 2030 & 2035 & 2040 \\
\hline $\begin{array}{l}\text { Arithmetisches Mittel } 1000 \text { günstigsten Spotpreise in } \\
€_{2017} / \mathrm{MW}_{\mathrm{el}} \mathrm{C}\end{array}$ & 3,19 & 1,39 & $-1,68$ & $-8,98$ & $-14,39$ & $-18,76$ \\
\hline Anzahl Stunden mit negativen Spotpreisen & 114 & 173 & 272 & 555 & 1000 & 1620 \\
\hline $\mathrm{CO}_{2}$-Preis in $€_{2017} / \mathrm{MW}_{\mathrm{th}} \mathrm{h}$ & 5,8 & 20,2 & 55,0 & 130,0 & 209,4 & 337,2 \\
\hline
\end{tabular}

nen Anzahl an Vollbenutzungsstunden) errechnet und eine Differenz-Investitionskostenrechnung durchgeführt.

Für das Basisszenario wurde die Entwicklung der EEGUmlage anhand von Literaturangaben abgeschätzt (Haller 2020). Bei den Netznutzungsentgelten wird der Arbeitspreis der Mittelspannung eines durchschnittlichen deutschen Stadtwerkes unter $2500 \mathrm{~h}$ in Höhe von $33,0 €_{2017} / \mathrm{MW}_{\text {el }} \mathrm{h}$ verwendet (SWHD Netze GmbH 2017). Darüber hinaus wird zunächst angenommen, dass über den gesamten Investitionszeitraum keine Kosten für $\mathrm{CO}_{2}$-Emissionen beim Erdgaseinsatz anfallen sowie das die jährliche Betriebszeit 1000 Vollbenutzungsstunden (VBS) beträgt. Alle weiteren technoökonomischen Parameter sind in Tab. 3 dargestellt.

Im Anschluss an den ökonomischen Vergleich der einzelnen Maßnahmen werden in einer Sensitivitätsanalyse für zwei ausgewählte Maßnahmen der Einfluss der Annahmen bezüglich des Zinses, der VBS und der Erdgasbezugskosten (ohne $\mathrm{CO}_{2}$-Kosten) einzeln untersucht.

\subsection{Rechtliche Analyse}

Innerhalb der rechtlichen Analyse wird unter anderem die Vereinbarkeit der Maßnahmen mit höherrangigem Recht, also dem Recht der Europäischen Union und dem Verfassungsrecht untersucht. Darüber hinaus wird analysiert, wie komplex die Maßnahmenumsetzung ist, sprich wie umfangreich die Eingriffe in den Rechtsrahmen sind und wessen Gesetzgebungskompetenz berührt ist. Die Analyse begrenzt sich dabei auf die oben ausgewählten Maßnahmen für eine PtH-Anlage im Netz eines Stadtwerkes.

\subsection{Analyse der Verteilungsgerechtigkeit}

Zur Analyse der Verteilungsgerechtigkeit werden die ausgewählten Maßnahmen qualitativ untereinander verglichen. Die Bewertung erfolgt dabei allgemeingültig aus systemischer Perspektive und nicht spezifisch auf die Situation der PtH-Anlage. Als Bewertungsparameter wird einerseits betrachtet, ob eine Maßnahme zu einer Verschiebung der Zahlung und zusätzlichen Belastung bereits stark belasteter Verbrauchergruppen führt, und ob die Zahlungsanpassung prinzipiell dem Verursachungsprinzip gerecht werden (siehe Agora Energiewende 2017). Erzeugungsseitig können die Maßnahmen indirekt ebenfalls Anpassungswirkungen entfalten, die die Gesamtsystemeffizienz erhöhen können und hinsichtlich der Verteilungswirkung berücksichtigt werden (siehe Ecofys 2014). Hierbei werden die Folgenden Aspekte besonders geprüft:

- Verschiebewirkung:

- Wer übernimmt zusätzliche Zahlungen?

- Zahlen aktuell stärker belastete Konsumenten mehr?

- Verursachungsgerechtigkeit

- Ist eine Mehr-/Minderbelastung einzelner berechtigt, da sie verstärkt profitieren?

- Sind Zahlende in der Lage, durch ihr Verhalten sich der neuen Regelung anzupassen?

Negativ wird dabei bewertet, wenn eine Maßnahme bereits stark betroffene Gruppen weiter belastet, d.h. die Verursachungsgerechtigkeit nicht gegeben ist. Positiv wird bewertet, wenn eine Maßnahme Verursachungsgerechtigkeit mit Mehrbelastung verbindet und die Zahlenden durch Verhaltensänderung sich der neuen Situation anpassen können.

\subsection{Synthese}

Abschließend wird auf Basis der Ergebnisse eine Priorisierung der einzelnen Maßnahmen durchgeführt. Hierzu wird auf die Rangfolge der Maßnahmen je Bewertungsperspektive zurückgegriffen. So ergibt sich die Rangfolge der ökonomischen Analyse anhand der Kapitalwerte der Differenzinvestitionen (Differenz-Kapitalwert). Bei der Analyse aus rechtlicher Perspektive und hinsichtlich der Verteilungsgerechtigkeit wird auf die qualitative Priorisierung der Maß- 
Tab. 3 Technoökonomische Parameter der Investitionsrechnung

\begin{tabular}{|c|c|c|}
\hline Name & Wert & Einheit \\
\hline Thermische Leistung PtH & 19,6 & $\mathrm{MW}_{\mathrm{th}}$ \\
\hline Thermischer Wirkungsgrad $\mathrm{PtH}^{\mathrm{a}}$ & 0,98 & $\mathrm{MW}_{\mathrm{th}} / \mathrm{MW}_{\mathrm{el}}$ \\
\hline Spez. Investitionsausgaben (inkl. Netzanschluss) $\mathrm{PtH}^{\mathrm{b}}$ & 140.627 & $€ / \mathrm{MW}_{\mathrm{el}}$ \\
\hline Kalkulatorische Lebensdauer PtH & 20 & a \\
\hline Jährliche Betriebs- und Wartungskosten PtH & 1000 & $€ /\left(\mathrm{MW}_{\mathrm{el}} * \mathrm{a}\right)$ \\
\hline Jährliches Leistungs-Netznutzungsentgelt ${ }^{\mathrm{c}}$ & 12.520 & $€ /\left(\mathrm{MW}_{\mathrm{el}} * \mathrm{a}\right)$ \\
\hline Stromnebenkosten (exkl. NE und EEG-Umlage) ${ }^{\mathrm{d}}$ & 23,80 & $€ / \mathrm{MW}_{\mathrm{el}} \mathrm{h}$ \\
\hline Spezifischer Emissionsfaktor Erdgas ${ }^{\mathrm{e}}$ & 0,201 & $\mathrm{t}_{\mathrm{CO} 2} / \mathrm{MW}_{\mathrm{th}} \mathrm{h}$ \\
\hline Thermischer Wirkungsgrad BHKW & 0,41 & $\mathrm{MW}_{\mathrm{th}} / \mathrm{MW}_{\text {th }}$ \\
\hline Elektrischer Wirkungsgrad BHKW & 0,38 & $\mathrm{MW}_{\mathrm{el}} / \mathrm{MW}_{\text {th }}$ \\
\hline Bezugskosten Erdgas (exkl. Gassteuer, $\mathrm{CO}_{2}$-Kosten) ${ }^{\mathrm{d}}$ & 21,43 & $€ / \mathrm{MW}_{\text {th }}$ \\
\hline
\end{tabular}

Alle Preisangaben netto und in $€_{2017}$

${ }^{\mathrm{a}}$ Götz et al. (2013, S. 6)

bEller (2015, S. $170 \mathrm{ff}$.)

'SWHD Netze GmbH (2017)

${ }^{\mathrm{d} B N e t z A}$ und BKartA (2017, S. 226, 384)

'UBA (2019, S. 16; eigene Annahmen) nahmen untereinander zurückgegriffen. Die abschließende Bewertung erfolgt durch den Vergleich der Summe ihrer Rankings.

\section{Bewertung und Umsetzungsempfehlungen}

Im Folgenden werden zunächst die Ergebnisse der ökonomischen und der rechtlichen Bewertung dargestellt. Im Anschluss erfolgt die Bewertung der Verteilungsgerechtigkeit aus systemischer Perspektive. Eine Synthese der Ergebnisse folgt in Abschn. 6.

\subsection{Ergebnisse der ökonomischen Analyse}

Abb. 3 stellt die Kapitalstruktur der Differenzinvestition, unter Einbezug des bestehenden BHKWs, für die einzelnen Maßnahmen dar. Es lässt sich feststellen, dass im Basisszenario in keiner einzelnen Periode ein Gewinn durch den Einsatz der PtH-Anlage mit 1000 VBS erzielt werden kann. Das BHKW erreicht hier noch im Jahr 2036 in einer einzelwirtschaftlichen Betrachtung, das heißt unter Einbezug der Fernwärmeerlöse, ein Gewinn in den 1000 günstigsten Stunden. Insgesamt ergibt sich für die Investition in eine PtH-Anlage unter den hier getroffenen Annahmen im Status Quo ein Kapitalwert von rund -16,87 Mio. $€_{2017}$. Sie ist also aus ökonomischer Sicht höchst unprofitabel.

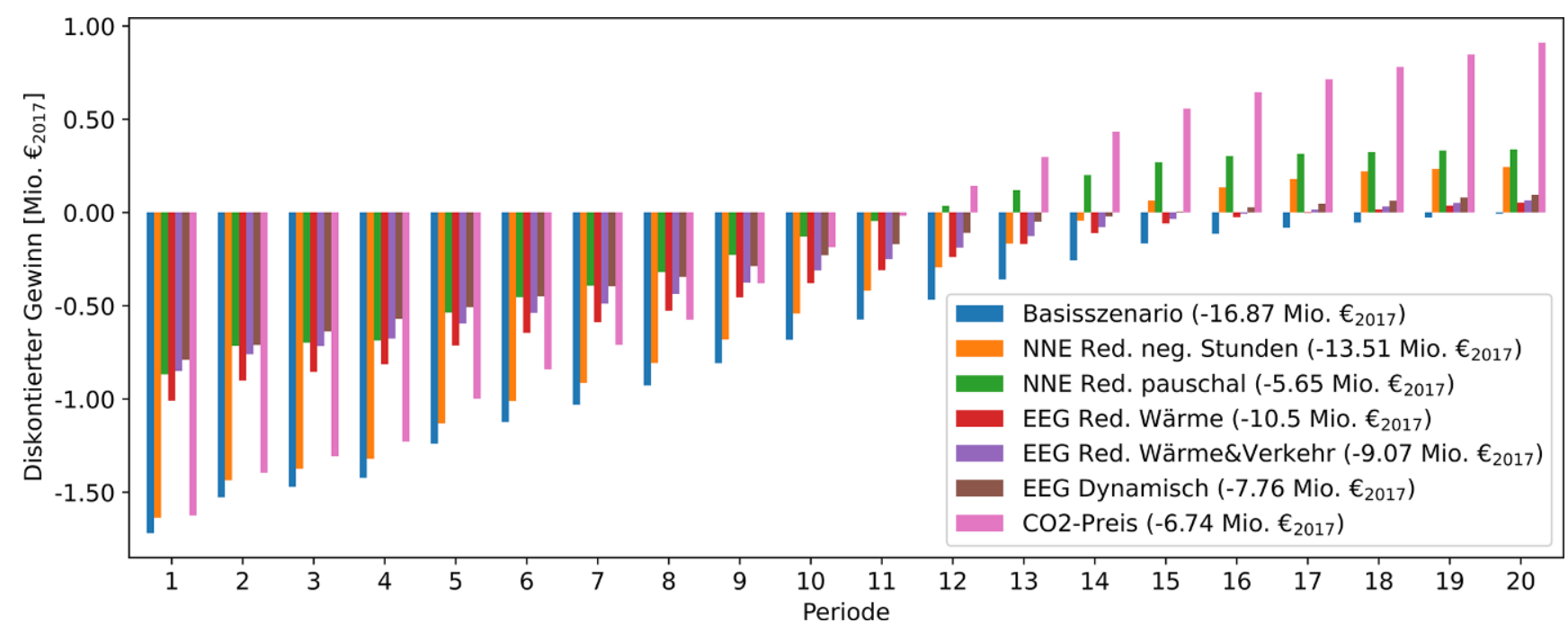

Abb. 3 Kapitalstruktur der Differenzinvestition für die analysierten Maßnahmen, Kapitalwerte in Klammern (Investitionsausgabe in Höhe von $-2.812 .540 €_{2017}$ nicht dargestellt, da für alle Szenarien gleich) 
Bei einer Befreiung des arbeitsbezogenen Netzentgeltes für Stunden mit negativen Strompreisen erzielt die PtH-Anlage ab 2031 einen jährlichen Gewinn. Jedoch führt der erst späte Anstieg negativer Stunden zum Ende der Lebensdauer dazu, dass diese Gewinne aufgrund des Zinseffektes den Kapitalwert mit rund 3 Mio. $€_{2017}$ nur leicht verbessern. Im Gegensatz dazu führt eine vollständige Befreiung von den Netznutzungsentgelten dazu, dass die Strombezugskosten der PtH-Anlage bereits am Anfang des Investitionszeitraumes stark gesenkt werden. Dadurch wird die Rentabilität der Differenzinvestition im Vergleich zum Status Quo um über 10 Mio. $€_{2017}$ gesteigert.

Eine breitere Wälzung der EEG-Umlage führt ebenfalls zu einer sofortigen Senkung der Strombezugskosten und somit zu einer Erhöhung der Rentabilität. Dieser Effekt nimmt jedoch vergleichsweise im Zeitverlauf ab, da insgesamt sinkende EEG-Kosten unterstellt werden. Während das Einbeziehen des Wärmesektors dabei die EEG-Umlage stark reduziert und den Kapitalwert um über 6 Mio. $€_{2017}$ erhöht, fällt der Einfluss des zusätzlichen Einbezugs des Verkehrssektors mit zzgl. 1 Mio. $€_{2017}$ eher gering aus. Die Dynamisierung der EEG-Umlage ergibt für die 1000 günstigsten Stunden des Jahres im Mittel eine Umlage von rund $18,91 €_{2017} / \mathrm{MW}_{\mathrm{el}} \mathrm{h}$ im Jahr 2017, womit sie diese von Anfang an stärker reduziert als bei den vorgestellten Wälzungsoptionen. Darüber hinaus fällt sie über den Analysezeitraum aufgrund sinkender Börsenstrompreise schneller ab, sodass sie im Jahr 2030 bei nur noch rund $3,70 €_{2017} / \mathrm{MW}_{\text {el }} \mathrm{h}$ verglichen mit $12,94 €_{2017} / \mathrm{MW}_{\text {el }} \mathrm{h}$ bei Wälzung auf Wärme- und Verkehrssektor liegt. Verglichen mit dem Status Quo wird die Investition damit über 9 Mio. $€_{2017}$ rentabler und somit besser als bei einer breiteren Wälzung der EEG-Umlage.

Im Unterschied zu den anderen Maßnahmen führt die Einführung eines sektorenübergreifenden $\mathrm{CO}_{2}$-Preises nicht zu einer direkten Steigerung der Rentabilität der PtH-Anlage, sondern zu einer Erhöhung der Opportunitätskosten durch die Verteuerung des Erdgaseinsatzes im BHKW. Darüber hinaus ist deren ökonomische Auswirkung zunächst gering und steigt lediglich im Zeitverlauf immer stärker an. Dies liegt jedoch mitunter auch am gewählten $\mathrm{CO}_{2}-$ Preispfad, welcher anfangs vergleichsweise geringe Preise aufweist und somit den Betrieb des BHKWs zunächst nur langsam verteuert. Jedoch kann bereits ab 2022, bei einem $\mathrm{CO}_{2}$-Preis von $32,50 €_{2017} / \mathrm{t}_{\mathrm{CO} 2}$, das $\mathrm{BHKW}$ bei einer einzelwirtschaftlichen Betrachtung in den 1000 günstigsten Stunden nicht mehr rentabel betrieben werden. Insgesamt ergibt sich damit ein Kapitalwert von rund -6,74 Mio. $€_{2017}$, wodurch diese aus ökonomischer Perspektive geringfügig schlechter zu bewerten ist als die pauschale Befreiung aller Netzentgelte.

Unter den getroffenen Annahmen ist die Investition in eine PtH-Anlage bei Umsetzung einer einzelnen hier ausgewählten Maßnahme in keinem Fall rentabel. Da solche Maßnahmen, welche unterschiedliche einzelne Kostenbestandteile (NNE, EEG-Umlage, $\mathrm{CO}_{2}$-Preis) betreffen, jedoch untereinander nicht konkurrieren, kann durch die Umsetzung mehrerer Maßnahmen eine erhebliche Steigerung des Kapitalwertes erreicht und die Investition insgesamt vorteilhaft werden.

Die Ergebnisse der Sensitivitätsanalyse für die Dynamisierung der EEG-Umlage (links) und die Einführung eines sektorenübergreifenden $\mathrm{CO}_{2}$-Preises (rechts) sind in Abb. 4 dargestellt. In beiden Szenarien führt ein Anstieg der Erdgasbezugskosten (ohne $\mathrm{CO}_{2}$-Kosten) des BHKWs um $10 \%$ zu einer Verbesserung des Kapitalwertes von rund 1,26 Mio. $€_{2017}$. Bei einem Anstieg der Erdgasbezugskosten auf ungefähr $34 €_{2017} / \mathrm{MW}_{\text {th }}$ h wird damit die Investition in die PtH-Anlage unter den hier getroffenen Annahmen insgesamt rentabel. Ein Anstieg der Vollbenutzungsstunden führt hingegen zu einem Anstieg der mittleren Strombezugskosten und dadurch zu einer sinkenden Rentabilität. Dabei ist der Kapitalwert bei einer Dynamisierung der EEG-Umlage wesentlich sensitiver, da hier nicht nur der
Abb. 4 Ergebnisse der Sensitivitätsanalyse für eine Dynamisierung der EEG-Umlage (links) und die Einführung eines sektorenübergreifenden $\mathrm{CO}_{2}$-Preises (rechts) bei einzelner Variation der Parameter

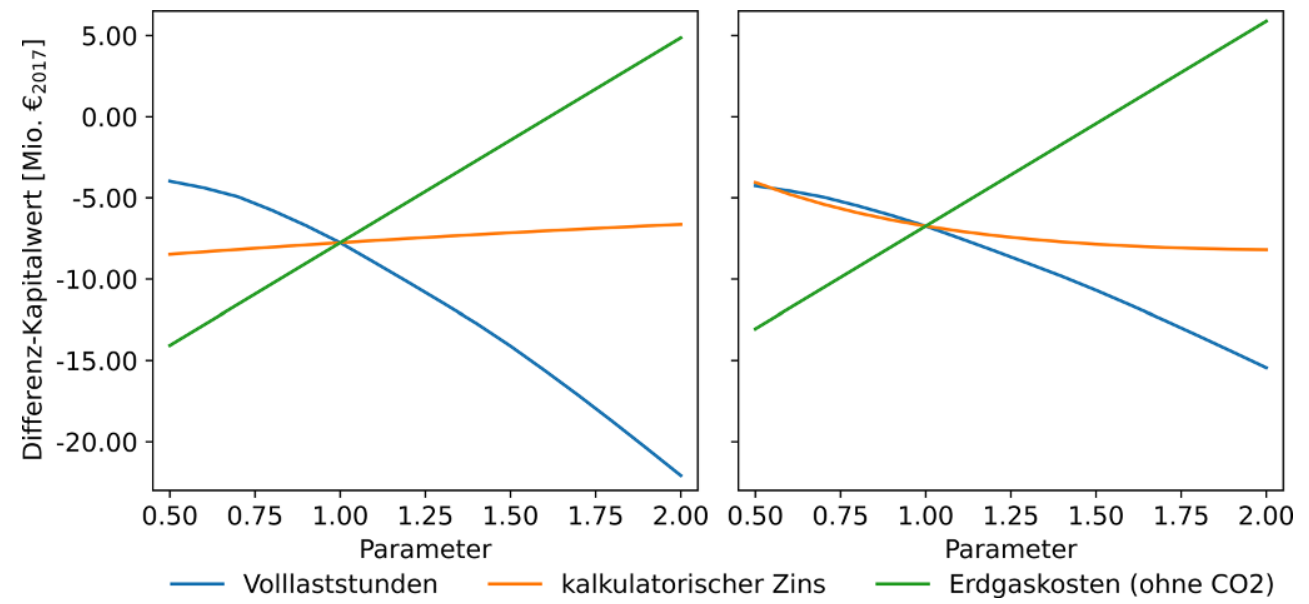


mittlere Spot-Strompreis, sondern auch die EEG-Umlage stark abhängig von den Vollbenutzungsstunden ist. Eine Verdopplung der Anzahl an Vollbenutzungsstunden auf 2000 führt demnach zu einer Senkung des Kapitalwerts um $184 \%$ während dieser sich bei Einführung ersterer Maßnahme nur um $134 \%$ verringert. Im Gegensatz dazu wirkt sich ein Anstieg der kalkulatorischen Verzinsung leicht positiv auf den Kapitalwert bei einer Dynamisierung der EEG-Umlage jedoch nicht bei Einführung eines sektorenübergreifenden $\mathrm{CO}_{2}$-Preises aus. Dieser Unterschied wird mit Blick auf die Kapitalstruktur der Investitionsalternativen in Abb. 3 ersichtlich, nach der bei letzterer Maßnahme zunächst große Verluste und zum Ende hin große Gewinne erzielt werden, welche jedoch bei höherer Verzinsung stärker diskontiert werden. Bei einer Dynamisierung der EEG-Umlage verbleiben die Gewinne durch den Betrieb der PtH-Anlage bis zum Ende derer Lebensdauer auf niedrigem Niveau, jedoch können die Kosten insbesondere in den ersten Betriebsjahren stark gesenkt werden.

\subsection{Ergebnisse der rechtlichen Analyse}

Die rechtliche Analyse konzentriert sich im Folgenden auf die vier oben genannten Maßnahmen. Die Sub-Maßnahmen werden nicht abgegrenzt, da sie rechtlich äquivalent zu bewerten sind.

Eine Erweiterung der Netzentgeltbefreiung, die bisher in $\S 118$ Abs. 6 EnWG eine Rückverstromung voraussetzt, kann durch die Aufnahme eines weiteren Ausnahmetatbestands in $\S 118$ EnWG erfolgen. Im Lichte des Art. 3 Grundgesetz (GG) und dem Verursachungsprinzip des $\S 21$ EnWG für die Netzentgeltberechnung sollte eine technologieneutrale Ausgestaltung der Maßnahme gewählt werden. Aufgrund der europäischen Beihilferegelungen der Art. 107, 108 des Vertrages über die Arbeitsweise der Europäischen Union (AEUV) ist die Anknüpfung an Stunden mit negativen Börsenstrompreisen vorzugswürdig. Eine Reduzierung kann gegenüber einer Befreiung leichter gerechtfertigt werden (Europäische Kommission 2018). Wird eine Umlage zum Ausgleich der geringeren Einnahmen erhoben, wie dies bei $\S 19$ Abs. 2 StromNEV der Fall ist, ist eine neuerliche Entscheidung der Europäischen Kommission zur Vereinbarkeit der Regelung mit dem Beihilferecht auf Basis von Art. 107, 108 AEUV zu erwarten. Erforderlich ist ein Bundesgesetz zur Änderung des EnWG. Es ist insofern eine einfache Mehrheit der abgegebenen Stimmen des Bundestages erforderlich, Art. 42 Abs. 2 Satz 1 GG.

Nicht unerhebliche Anpassungen des Rechtsrahmens erforderte eine Dynamisierung der EEG-Umlage. Die konkrete Ausgestaltung muss eine Vereinbarkeit mit den verfassungsrechtlichen Vorgaben gewährleisten. Aufgrund des Gleichheitsgrundsatzes des Art. 3 Abs. 1 GG bedarf eine Ungleichbehandlung wesentlich gleicher Lebenssachver- halte eines einleuchtenden (sachlichen) Grundes (BVerfG 1980, S. 88 f.). Als sachliche Rechtfertigung der im Vergleich höheren EEG-Umlage der unflexiblen Verbraucher kommt die Netz- und Systemdienlichkeit des Anlageneinsatzes zur Integration von Strom aus erneuerbaren Energien in Betracht. Ein aus der unterschiedlichen Höhe der EEGUmlage resultierender Eingriff in die Berufsfreiheit des Art. 12 GG lässt sich grundsätzlich verfassungsrechtlich rechtfertigen, da hierfür hinreichende Gründe des Allgemeinwohls (BVerfG 1985, S. 196) genügen. Das verfolgte Ziel des Klimaschutzes, das durch die Integration erneuerbarer Energien gefördert werden soll, hat sogar durch Art. 20a GG, dem Schutz der natürlichen Lebensgrundlagen, Verfassungsrang (Scholz in Maunz und Dürig 2020, Art. 20a Rn. 5). Mit Blick auf die Finanzverfassung muss die Ausgestaltung so gewählt werden, dass der Charakter einer Preisregelung zwischen Privatrechtssubjekten erhalten bleibt (Däuper und Lachmann 2018, S. 6). Die Grundstruktur der EEG-Umlage sollte zudem beibehalten werden, um nicht den Tatbestand der Beihilfe zu erfüllen (Art. 107 AEUV). $\mathrm{Zu}$ berücksichtigen ist, dass Einnahmen aus der $\mathrm{CO}_{2}$-Bepreisung ab 2021 zur Reduzierung der EEG-Umlage insgesamt verwendet werden sollen (Bundesregierung 2019b, S. 29), was die Höhe der Umlage künftig reduziert. Angepasst werden müssen § 60 EEG 2017 und im Rahmen der Erneuerbare-Energien-Verordnung (BGBl. 2015 I, S. 146) die Vorschriften zur Ermittlung der EEG-Umlage.

In der Ausgestaltungsvariante der Verbreiterung der Umlagebasis zur Reduzierung der EEG-Umlage im Stromsektor muss gleichfalls ein besonderes Augenmerk auf die Vereinbarkeit mit den Beihilferegelungen des Rechts der Europäischen Union, Art. 107 AEUV und eine mögliche neue Entscheidung der Kommission gelegt werden. Maßgeblich ist, ob es sich trotz der Anpassungen nicht um staatliche oder aus staatlichen Mitteln gewährte Beihilfen handelt. Auch wenn die Zuständigkeit der Übertragungsnetzbetreiber aus § 60 Abs. 1 EEG 2017 für die Umverteilung der Mittel erhalten bleibt, wird eine erheblich größere gesetzliche Steuerungswirkung erzielt, da Mittel sektoren- und energieträgerübergreifend erhoben werden und dem Stromsektor zugutekommen (Däuper und Lachmann 2018, S. 6). Eine Bejahung des Beihilfetatbestands durch die Kommission ist jedenfalls nicht fernliegend. Auf der Ebene der deutschen Verfassung ist zu prüfen, ob es sich bei einer Erweiterung der EEG-Umlage weiterhin um eine Preisregelung zwischen Privatrechtssubjekten und nicht um eine Sonderabgabe handelt. Vorausgesetzt ist, dass - wie dies bei der bestehenden EEG-Umlage vom BGH bejaht wird - keine Aufkommenswirkung zugunsten der öffentlichen Hand besteht (BGH 2014). Formal ist dies anzunehmen, sofern die Mittel nicht über einen Sonderfonds verwaltet werden, sondern ausschließlich Bestandteil von Leistungs-, Abnahme- und Zahlungsverpflichtungen zwischen Rechtssubjek- 
ten des Privatrechts sind (BGH 2014, Rn. 16). Werden in den Sektoren Wärme und Verkehr jedoch fossile Energieträger eingesetzt, besteht zu den Akteuren des Stromsektors gerade kein Vertragsverhältnis, sodass Anhaltspunkte für einen Formmissbrauch zu bejahen sein könnten (Däuper und Lachmann 2018, S. 6f.). Die verfassungsrechtlichen Anforderungen an eine Sonderabgabe sind jedoch ebenfalls nicht erfüllt. Vorausgesetzt ist, dass die Umlage gruppennützig verwendet wird (BVerfG 2014). Es profitiert von der Umlage ausschließlich der Stromsektor und nicht die ebenfalls umlagebelasteten Verbraucher des Wärme- und Verkehrssektors.

Das Recht der Europäischen Union gewährt den Mitgliedstaaten für die Bepreisung von $\mathrm{CO}_{2}$ Emissionen einen weiten Gestaltungsspielraum (Erwägungsgrund 23 der Emissionshandelsrichtlinie Europäische Union 2003). Mit dem Brennstoffemissionshandelsgesetz (BEHG) ist der Bundesgesetzgeber im Dezember 2019 (BGBl. 2019 I, S. 2728) einen Schritt auf die sektorenübergreifende $\mathrm{CO}_{2}{ }^{-}$ Bepreisung zugegangen und hat in Ergänzung zum europäischen Emissionshandel (EU-ETS) ein nationales Bepreisungssystem für Treibhausgasemissionen aus Brennstoffen vorbereitet. Die Einführung einer Bepreisung muss sich in den engen Rahmen der Finanzverfassung als Teil des Grundgesetzes einpassen. Die Erhebung einer neuen, in der Finanzverfassung nicht vorgesehenen Steuerart bedarf einer Änderung des Grundgesetzes mit einer Zweidrittelmehrheit. Diese Änderung ist bei der Erhebung einer Steuer auf den Ausstoß von $\mathrm{CO}_{2}$ notwendig, da sich dieses Instrument keiner der in der Finanzverfassung vorgesehenen Steuerarten zuordnen lässt. Eine Ausgestaltung der $\mathrm{CO}_{2}$-Bepreisung als Vorteilsabschöpfungsabgabe, bedarf lauft BVerfG eines „,knapp definierte[n] Gut[s]“, das ,,mengenmäßig begrenzt ${ }^{\text {“ }}$ wird (BVerfG 2018, Rn. 34). Die durch das Brennstoffemissionshandelsgesetz (BEHG) vorgesehenen Zertifikate haben trotz der Bezeichnung als Emissionshandel während einer mehrjährigen Einführungsphase keinen Cap, also keine verbindliche Emissionsobergrenze. Daher hat die im BEHG vorgesehene Bepreisung starke Elemente einer Steuer und wirkt zunächst vergleichbar. Dies wirft Fragen der Verfassungskonformität des gewählten Instruments auf, das als einfaches Parlamentsgesetz verabschiedet wurde. Den verfassungsrechtlichen Bedenken kann allenfalls damit begegnet werden, dass es zur Einführung eines Caps einer Übergangszeit bedarf, wobei diese dann wohl kürzer sein müsste als die derzeit im BEHG vorgesehene. Positiv zu bewerten ist, dass perspektivisch die Möglichkeit bestehen, den nationalen und den europäischen Emissionshandel zusammenzuführen. Als Steuer ließe sich die Bepreisung ohne die Notwendigkeit einer Änderung der Finanzverfassung mittels eines einfachen Parlamentsgesetzes ausgestalten, indem die bestehenden Energiesteuern (Verbrauchssteu- er) anhand des $\mathrm{CO}_{2}$-Gehalts des Energieträgers gewichtet werden (IKEM und Rodi 2019).

\subsection{Bewertung der Verteilungsgerechtigkeit}

Die Verteilungsgerechtigkeit der Maßnahmen ist sehr unterschiedlich zu bewerten. So führt eine Minderung der Netzentgelte für die PtH-Anlage dazu, dass diejenigen Verbraucher, die bereits anteilig die höchste Last tragen, weitere Zahlungen übernehmen müssen. Dieses gilt für die komplett Befreiung von den Netzentgelten mehr als für die Anteilige, da die Gesamtsumme höher ist. Eine Veränderung auf Erzeugungsseite ist nicht zu erwarten. Ebenso ist in beiden Fällen eine Verursachungsgerechtigkeit für die Mehrbelastung, speziell im pauschalen Fall nicht zu identifizieren.

Die Wälzung der EEG-Umlage auf weitere Sektoren führt zunächst zu einer Reduktion der strombezogenen EEG-Umlage und einer Entlastung aller Stromverbraucher. Durch die Vergünstigung des Stromes unterstützt die Maßnahme einen verstärkten Erneuerbaren Stromeinsatz im Sinne der Sektorenkopplung und nivelliert den Einsatz von strombasierten Technologien hinsichtlich der Abgabenlast (Agora Energiewende 2018, S. 13). Es ist aber zu bemerken, dass besonders der Haushaltssektor verstärkt belastet würde, während bereist begünstigte Verbraucher tendenziell weniger beitragen müssen (Gährs et al. 2017, S. 16). Eine Rückwirkung auf die Nachfrageseite, d.h. eine Verbesserung der Gesamtsystemeffizienz ist nicht $\mathrm{zu}$ erwarten, wobei erhöhte Kosten im Wärmesektor eine Verbrauchsanpassung ergeben könnten. Insofern besteht hier eine Verursachungsgerechtigkeit hinsichtlich des Energieeinsatzes durch die Endkunden, der mittelfristig zu einer Technologieanpassung führen kann. Hierbei sei allerdings auf das Mieter-Vermieter-Dilemma hingewiesen, das diesem im Wege steht. Insgesamt ist die Maßnahme allerdings besser zu bewerten als die Reduktion der Netzentgelte.

Bei der Dynamisierung der EEG-Umlage stellen lediglich einzelne Aspekte eine Verbesserung gegenüber einer Wälzung dar. Zunächst stellt die Dynamisierung ein erhöhtes Risiko für Endkunden (an die sie weitergegeben wird) dar, welches negativ zu bewerten ist. Allerdings ist davon auszugehen, dass die Dynamisierung zu einer Anpassung des (zeitlichen) Verbrauchsverhaltens führt. Dies resultiert in einem verbesserten Kraftwerkseinsatz auf Erzeugungsseite und ebenso einer Reduktion der EEG-Umlage durch die mittelfristig zu erwartenden steigenden Preisen der günstigsten Stunden und der damit korrelierenden Erhöhung des Marktwertes von EE-Strom (Ecofys 2014, S. 21). Hinsichtlich der Verursachungsgerechtigkeit ist festzustellen, dass Preisschwankungen primär durch die Erneuerbaren Energien hervorgerufen werden und nicht durch die (verstärkt) belasteten Endkunden. Positiv ist aber zu be- 
werten, dass eine Verhaltensanpassung eine Reduktion der Kosten verursachen kann.

Abschließend ist die $\mathrm{CO}_{2}$-Bepreisung aus Perspektive der Verteilungs- und Verursachungsgerechtigkeit im Vergleich am besten zu bewerten. Dies ist darin begründet, dass die zusätzlichen Zahlungen, die durch Konsumierende zu leisten sind, sehr direkt mit ihren Verbräuchen gekoppelt sind. Zu beachten ist zwar, dass es sich um eine ,zusätzliche“ Zahlung handelt, allerdings ist hierbei zu bedenken, dass eine $\mathrm{CO}_{2}$-Bepreisung lediglich eine Integration von externen Kosten darstellt. Diese müssen langfristig alternativ anderweitig getragen werden, sodass dies nicht als negativ zu bewerten ist. Zudem ist davon auszugehen, dass eine $\mathrm{CO}_{2}$-Bepreisung ebenfalls, durch die Anpassung des Konsumverhaltens der Verbraucher, eine Lenkungswirkung auf die Erzeugungsseite verursacht. Dies führt mittelfristig zu einem effektiveren Energiesystem, dass insgesamt (d.h. unter Einbezug externer Kosten) kostengünstiger ist, wovon die Verbraucher zusätzlich profitieren. Darüber hinaus entstehen auf staatlicher Seite Erlöse, welche an Konsumierende zurückerstattet werden können. Dies könnte beispielsweise über die Senkung der Stromsteuer oder EEGUmlage geschehen (Frondel 2020), welche sich zusätzlich ökonomisch positiv auf den Betrieb der PtH-Anlage auswirkt.

\subsection{Synthese}

Hinsichtlich der Verbreiterung der EEG-Umlage durch die Einbeziehung der Sektoren Wärme und Verkehr bestehen nicht nur verfassungsrechtliche Unsicherheiten, sowie die eines Notifizierungsverfahrens der Europäischen Kommission, sondern auch Schwächen aus ökonomischer Perspektive. Hinsichtlich der Verteilungsgerechtigkeit ist die Maßnahme ebenfalls vergleichsweise schlecht zu bewerten, sodass die sektorenübergreifende Wälzung an letzter Stelle priorisiert wird.

Nicht deutlich besser schneidet die Reduktion der Netzentgelte ab. Hierbei ist festzustellen, dass diese sich aus rechtlicher Sicht relativ einfach umsetzen lässt, sofern die Vorschrift technologieneutral ausgestaltet wird und beispielsweise an den netz- und systemdienlichen Einsatz von Letztverbrauchern anknüpft, wobei eine Reduzierung gegenüber einer Befreiung vorzugswürdig ist. Die Vorzugswürdigkeit der Reduktion gegenüber der Befreiung gilt auch für die Verteilungsgerechtigkeit, wobei die Maßnahme insgesamt an letzter Stelle gerankt wird. Aus ökonomischer Sicht ist allerdings gerade die vollständige Befreiung besonders positiv zu bewerten, welche aber kaum einem netz- und systemdienlichen Einsatz entsprechen bzw. diesen fördern dürfte. Die Reduktion schneidet aus ökonomischer Sicht hingegen am schlechtesten ab, sodass die Maßnahme insgesamt an dritte Position gesetzt wird.

Die Dynamisierung der EEG-Umlage ist aus rechtlicher Perspektive gut umsetzbar, wobei die Grundstruktur der EEG-Umlage beibehalten werden sollte. Aus ökonomischer Sicht der PtH-Anlage ist die Dynamisierung aufgrund der Flexibilität der Anlage ebenfalls vorteilhaft und aus systemischer Perspektive kann sogar auf eine Verbesserung der Gesamtsystemeffizienz und eine Reduktion der Abgabe an sich gehofft werden. Die Maßnahme wird daher insgesamt an zweiter Stelle empfohlen.

Dominant wirkt aus jeder Bewertungsperspektive die Einführung einer sektorenübergreifenden $\mathrm{CO}_{2}$-Bepreisung. Diese ist aus rechtlicher Sicht zwar vergleichsweise komplex. Dennoch ist aber bei einer Gewichtung der Energiesteuern oder einer Ausgestaltung als Emissionshandel lediglich das Bundesrecht betroffen, sodass eine Umsetzung auf der Ebene des einfachen Bundesrechts möglich ist. Aus ökonomischer Perspektive ist bei dem angenommenen Preispfad die Maßnahme an zweiter Stelle bewertet, wobei die fehlende Dominanz dem Vergleichsweise günstigen $\mathrm{CO}_{2}$-Preispfad geschuldet ist. Aus systemischer Perspektive ist hier die höchste Verursachungsgerechtigkeit zu erreichen, sowie eine steigende Effizienz des Gesamtsystems zu erwarten. Der Maßnahme ist daher aus rechtswissenschaftlicher, ökonomischer und systemischer Perspektive nach Einschätzung der Autor*innen dieser Maßnahme klar der Vorzug zu gegeben werden.

\section{Zusammenfassung und Ausblick}

Die Arbeit stellt zunächst den aktuellen Rechtsrahmen bezüglich PtH dar und leitet im Folgenden im Rahmen eines zweistufigen Verfahrens vier Maßnahmen aus der Literatur ab, die aus ökonomischer und rechtlicher Perspektive sowie hinsichtlich der Verteilungsgerechtigkeit untersucht werden. Hierbei ergibt sich eine klare Dominanz für die Einführung einer sektorenübergreifenden $\mathrm{CO}_{2}$-Bepreisung Jedoch bedarf es weiterer Maßnahmen, um eine betriebswirtschaftliche Rentabilität der PtH-Anlage unter den getroffenen Annahmen zu realisieren, welche möglicherweise auch durch Erlöse aus der $\mathrm{CO}_{2}$-Bepreisung finanziert werden könnten. Hierbei ist eine Dynamisierung der EEGUmlage eine gute Ergänzung. Eine Reduktion der Netzentgelte erscheint aus rechtlicher Sicht umsetzbar, ist aber aus einzelökonomischer Perspektive weniger sinnvoll. Die Befreiung der PtH-Anlage von Netzentgelten ist einzelökonomisch zwar sehr wirksam, aber rechtlich aufwendiger und vor dem Hintergrund der Verteilungsgerechtigkeit schwächer zu bewerten. Rechtlich kompliziert erscheint die Verbreiterung der EEG-Aufkommensbasis, welche eben- 
falls aus Verteilungsgerechtigkeitsaspekten und ökonomischer Perspektive nur niedrig zu priorisieren ist.

Methodisch verbindet die vorliegende Analyse qualitative und quantitative Elemente in einem interdisziplinären Ansatz. Hierbei war es notwendig, Einschränkungen hinsichtlich der Komplexität zu treffen und die Schnittstellen zwischen den Verfahren und Disziplinen passgenau zu gestalten. Als weiterer Forschungsbedarf wurde bei aus ökonomischer Perspektive festgestellt, dass eine Analyse der Rückkopplung von PtH-Nutzung und Strompreisen besonders bei steigender PtH-Durchdringung genauer zu analysieren ist. Dies gilt umso mehr, wenn eine Dynamisierung der EEG-Umlage eingeführt wird und somit der Anreiz für flexible Lasten weiter verstärkt wird. Diesbezüglich ist ebenfalls fraglich, inwieweit dies zu einem steigenden Marktwert von EE-Anlagen führt und damit zu einer Reduktion der EEG-Umlage. Diese Untersuchungen sind ebenfalls für eine quantitative Abschätzung der Verteilungsgerechtigkeit interessant. So könnten anhand eines ausdifferenzierten ökonomischen Fluss-Modells die Zahlungsflüsse genauer evaluiert und die qualitativen Elemente dieser Analyse um quantitative Aussagen ergänzt werden. Weiterer Forschungsbedarf aus rechtlicher Perspektive ergibt sich hinsichtlich einer grundsätzlichen Neuausrichtung des Energierechts in dessen Zentrum der Einsatz von Strom aus Erneuerbaren Energien steht. Wie gezeigt werden konnte, ist die $\mathrm{CO}_{2}$-Bepreisung hier ein erster, richtiger Schritt. Es bedarf allerdings eines Paradigmenwechsels, der in vielen Bereichen das bestehende Regel-Ausnahmeverhältnis umkehrt.

Förderung Der vorliegende Artikel ist auf Basis der Arbeiten des durch das Bundesministerium für Bildung und Forschung (BMBF) geförderten Kopernikus Projekts „Systemintegration“: Energiewende-Navigationssystem (ENavi) (Förderkennzeichen 03SFK4T0 und 03SFK4D0) entstanden.

\section{Literatur}

Agora Energiewende (2017) Neue Preismodelle für Energie. Grundlagen einer Reform der Entgelte, Steuern, Abgaben und Umlagen auf Strom und fossile Energieträger. Agora Energiewende, Berlin

Agora Energiewende (2018) Eine Neuordnung der Abgaben und Umlagen auf Strom, Wärme, Verkehr. Optionen für eine aufkommensneutrale $\mathrm{CO}_{2}$-Bepreisung. Agora Energiewende, Berlin

BCG, Prognos AG (2018) Klimapfade für Deutschland. Studie im Auftrag des BDI. BCG, Prognos AG, München, Hamburg, Basel, Berlin

BGH (2014) Urteil v. 25. Juni 2014 - VIII ZR 169/13, ZUR 2014, 562

BGH (2017) Beschluss v. 20. Juni 2017 - EnVR 24/16

BMU (2019) Klimaschutzplan 2050, 2. Aufl. BMU, Berlin

BNetzA, BKartA (2017) Monitoringbericht 2017. Bundesnetzagentur für Elektrizität, Gas, Telekommunikation, Post und Eisenbahnen; Bundeskartellamt, Bonn

BNetzA, BKartA (2019) Monitoringbericht 2019 - Kernaussagen. Bundesnetzagentur für Elektrizität, Gas, Telekommunikation, Post und Eisenbahnen; Bundeskartellamt, Bonn
Bundesregierung (2019a) Grundlage für $\mathrm{CO}_{2}$-Preis steht. In: Klimaschutz. https://www.bundesregierung.de/breg-de/themen/ klimaschutz/nationaler-emissionshandel-1684508. Zugegriffen: 1. Mai 2020

Bundesregierung (2019b) Klimaschutzprogramm 2030 der Bundesregierung zur Umsetzung des Klimaschutzplans 2050

BVerfG (1980) BVerfGE 55,72

BVerfG (1985) BVerfGE 71, 183

BVerfG (2014) BVerfGE 135, 155

BVerfG (2018) Beschluss 1 BvR 2864/13

Däuper O, Lachmann H-C (2018) Rechtliche Optionen für die Weiterentwicklung der EEG-Umlage und eine neue Finanzierung der Energiewende. Z Gesamt Recht Energiewirtsch 1/2:3-12

Dertinger A, Schill W-P (2019) Ansätze zur Umgestaltung von Abgaben und Umlagen auf Strom sowie Heiz- und Kraftstoffe. DIW Roundup 14:5

Doderer H, Steffensen S, Schäfer-Stradowsky S (2018) Power to heat - Eine Chance für die Energiewende. IKEM - Institut für Klimaschutz, Energie und Mobilität e.V, Berlin

Drünert S, Neuling U, Timmerberg S, Kaltschmitt M (2019) Powerto-X $(\mathrm{PtX})$ aus „Überschussstrom“ in Deutschland - Ökonomische Analyse. Z Energiewirtsch 43:173-191. https://doi.org/10. 1007/s12398-019-00256-7

Ecofys (2014) Der Spotmarktpreis als Index für eine dynamische EEGUmlage. Ecofys Germany GmbH, Berlin

Edenhofer O, Flachsland C, Kalkuhl M et al (2019) Optionen für eine $\mathrm{CO}_{2}$-Preisreform. Mercator Research Institute on Global Commons and Climate Change (MCC) $\mathrm{gGmbH}$, Berlin

Eller D (2015) Integration erneuerbarer Energien mit Power-to-Heat in Deutschland. Springer Vieweg, Wiesbaden

Europäische Kommission (2018) EU Kommission Beschluss Befreiung Netzentgelte Bandlast

Europäische Union (2003) Richtlinie 2003/87/EG des Europäischen Parlaments und des Rates vom 13. Oktober 2003 über ein System für den Handel mit Treibhausgasemissionszertifikaten in der Gemeinschaft und zur Änderung der Richtlinie 96/61/EG des Rates

Fiedler S, Zerzawy F, Sax A et al (2017) Alternative Finanzierungsoptionen für erneuerbare Energien im Kontext des Klimaschutzes und ihrer zunehmenden Bedeutung über den Stromsektor hinaus. Forum Ökologisch-Soziale Marktwirtschaft e. V., Berlin

Fraunhofer ISE (2020) Wege zu einem klimaneutralen Energiesystem - Die deutsche Energiewende im Kontext gesellschaftlicher Verhaltensweisen. Fraunhofer ISE, Freiburg

Fraunhofer IWES/IBP (2017) Wärmewende 2030. Schlüsseltechnologien zur Erreichung der mittel- und langfristigen Klimaschutzziele im Gebäudesektor. Studie im Auftrag von Agora Energiewende. Fraunhofer IWES/IBP, Berlin

Fraunhofer IWES, Stiftung Umweltrecht, Fraunhofer IFAM (2014) Power-to-Heat zur Integration von ansonsten abgeregeltem Strom aus Erneuerbaren Energien. Fraunhofer IWES, Stiftung Umweltrecht. Fraunhofer IFAM, Berlin

Frondel M (2020) $\mathrm{CO}_{2}$-Bepreisung in den Sektoren Verkehr und Wärme: Optionen für eine sozial ausgewogene Ausgestaltung. Z Energiewirtsch 44:1-14. https://doi.org/10.1007/s12398-020-00272-y

Frontier Economics BET (2016) Kosten und Nutzen einer Dynamisierung von Strompreiskomponenten als Mittel zur Flexibilisierung der Nachfrage. Frontier Economics Ltd, BET Büro für Energiewirtschaft und technische Planung $\mathrm{GmbH}$, London Berlin

Gährs S, Hirschl B, Aretz A (2017) Möglichkeiten zur Umgestaltung der EEG-Umlagebasis. Institut für ökologische Wirtschaftsforschung, Berlin

Ghosh T, Kochems J, Grosse B, Müller-Kirchenbauer J (2019) Modelling of imports and exports for the German electricity system. ENERDAY 2019-13th International Conference on Energy Economics and Technology, Dresden

Gils C, Dotzauer M, Acksel D et al (2016) Sektorenkopplung als Baustein der Energiewende. FVEE-Themen 
Götz M, Böttger D, Kondziella H, Bruckner T (2013) Economic potential of the ,power-to-heat" technology in the 50hertz control area

Haller M (2020) AGORA-EEG-Rechner_v3_5_4. https://www.agoraenergiewende.de/fileadmin2/Projekte/EEG-Rechner/Prognose_ 2020/AGORA-EEG-Rechner_v3_5_4.xlsx. Zugegriffen: 4. Mai 2020

Hilpert J (2015) Der Rechtsrahmen für Power-to-Heat-Anwendungen. Tagungsband der Dialogplattform des EFZN zum Thema „Erneuerbare erfolgreich integrieren durch Power to Heat"“. Bd. 33, S $128-135$

Hilpert S, Kaldemeyer C, Krien U et al (2018) The open energy modelling framework (oemof) - A new approach to facilitate open science in energy system modelling. Energy Strategy Rev 22:16-25. https://doi.org/10.1016/j.esr.2018.07.001

IKEM, Rodi M, Rodi M (2019) Verfassungsmäßigkeit des Entwurfs zum Brennstoffemissionshandelsgesetzes (BEHG-E). IKEM Institut für Klimaschutz, Energie und Mobilität e.V; Universität Greifswald, Berlin, Greifswald

IW (2018) Möglichkeiten einer $\mathrm{CO}_{2}$-Bepreisung im Wärmemarkt. Institut der deutschen Wirtschaft, Köln

Jansen M, Sager-Klauß C (2017) Das gekoppelte Energiesystem. E4tech. Fraunhofer IEE, London, Kassel

Maunz T, Dürig G (2020) Grundgesetz Kommentar, Werkstand: 90. EL
Reuster L, Runkel M, Zerzawy F et al (2017) Energiesteuerreform für Klimaschutz und Energiewende. Forum Ökologisch-Soziale Marktwirtschaft e. V., Berlin

Stiftung Umweltenergierecht (2016) Gutachten zu zuschaltbaren Lasten. Stiftung Umweltenergierecht; Fraunhofer Institut für Systemund Innovationsforschung (ISI), Würzburg, Karlsruhe

SWHD Netze GmbH (2017) Netznutzung Strom. Preisblätter der Stadtwerke Heidelberg Netze GmbH für die Netznutzung Strom (gültig ab 01.01.2017). https://www.swhd.de/de/SWH/Netze/ veroeffentlichungspflichten/Strom/Netzzugang/Strom-Netzent gelte/Preisblatt-Netzentgelte-Strom-2017.pdf. Zugegriffen: 29. Apr. 2020

UBA (2020) Energieverbrauch für fossile und erneuerbare Wärme. In: Umweltbundesamt. https://www.umweltbundesamt.de/daten/ energie/energieverbrauch-fuer-fossile-erneuerbare-waerme. Zugegriffen: 20. Mai 2020

UBA (2019) Entwicklung der spezifischen Kohlendioxid-Emissionen des deutschen Strommix in den Jahren 1990-2018. Umweltbundesamt, Dessau-Roßlau

Wietschel M, Plötz P, Klobasa M et al (2019) Sektorkopplung - Was ist darunter zu verstehen? Z Energiewirtsch 43:1-10. https://doi. org/10.1007/s12398-018-0241-3 